\title{
Oxidation of PAHs in water solutions by ultraviolet radiation combined with hydrogen peroxide
}

\author{
Stanisław Ledakowicz, Jacek S. Miller, ${ }^{\dagger}$ and Dorota Olejnik \\ Faculty of Process and Environmental Engineering, Technical University of Łódź \\ ul. Wólczańska 213, 90-924 Łódź, Poland
}

\begin{abstract}
The destruction of three polycyclic aromatic hydrocarbons (PAHs): benzo[a]pyrene, chrysene and fluorene in aqueous solution using advanced oxidation process $\mathrm{H}_{2} \mathrm{O}_{2} / \mathrm{UV}$ was investigated. The influence of $\mathrm{pH}$, initial hydrogen peroxide and radical scavenger concentrations on the reaction rate was studied. The oxidation reactions most rapidly run in neutral and acidic solution at optimal hydrogen peroxide concentration $(c a .0 .01 \mathrm{M})$. The degradation of benzo[a]pyrene and chrysene follows radical reaction, for fluorene the mechanism is not clear. The rate constants of the hydroxyl radicals and selected PAHs reaction were found to be $2.53 \times 10^{10}, 9.82 \times 10^{9}$ and $2.77 \times 10^{9} \mathrm{M}^{-1} \mathrm{~s}^{-1}$ for benzo[a]pyrene, chrysene and fluorene, respectively.
\end{abstract}

\section{INTRODUCTION}

PAHs are ubiquitous in the ecosphere. They were found in the atmosphere [1], water [2], soil [3] as well as plants [4] and animals [2]. Their presence in drinking water was also detected [5]. Natural events such as forest fires, and cardinal anthropogenic activity, and first of all uncompleted combustion of fossil fuels are the main sources of PAHs emission. From the atmosphere or soil they are easily moved to surface waters. Increased attention has been paid to these compounds due to the mutagenic, teratogenic and carcinogenic character of some of these molecules even in very small doses [6]. So PAHs constitute an important, hazardous group of water micropollutants. With regard of their very low aqueous solubility classical methods of water purification are ineffective. Only oxidation or UV radiation can destroy these contaminants to some extent, leaving sometimes toxic by-products [7]. It seems that this type of processes combined with biological treatment are the most promising methods of PAHs elimination from water environment [8].

Processes based on the formation of highly reactive hydroxyl radicals to act as an oxidant have been designated as advanced oxidation processes (AOPs). Among different possibilities hydroxyl radicals can be generated by photolysis of hydrogen peroxide. This technique was widely used for destruction of various harmful compounds e.g. phenol [9], pentachlorophenol [10], halogenoalkanes [11], pesticides [12], surfactants [13]. PAHs were also the object of studies in the $\mathrm{H}_{2} \mathrm{O}_{2}$ /UV system. Beltran et al. [14] widely investigated the dependence of initial hydrogen peroxide concentration and $\mathrm{pH}$ of reaction medium on the decomposition rate of acenaphthene, fluorene and phenanthrene in aqueous solutions. The influence of the hydroxyl radical scavenger-bicarbonate ion on reaction effectiveness was also studied. Surprisingly the presence of bicarbonate ion did not change the reaction course.

The kinetics of the degradation of the other PAHs set

† miller@wipos.p.lodz.pl were studied by Hautaniemi et al. [15]. The decomposition of naphthalene by $\mathrm{H}_{2} \mathrm{O}_{2}$ alone and with UV radiation was investigated by Tuhkanen [16]. The influence of various parameters on reaction rate and the rate constants was determined [14-16].

The aim of present work was to study the benzo[a]pyrene (BAP), chrysene (CHR) and fluorene (FLU) degradation in aqueous solution in the presence of hydrogen peroxide and UV radiation. The influence of the $\mathrm{pH}$ of reaction solution and the presence of radical scavenger, tert-butyl alcohol $(t-\mathrm{BuOH})$ was also investigated. Presented work is the second part of our study on application of different AOPs techniques for PAHs elimination from aqueous medium. The first part devoted to PAHs direct photolysis is now under preparation.

\section{MATERIALS AND METHODS}

The experiments were carried out in a $1 \mathrm{dm}^{3}$ thermostated glass reactor equipped with the central well for immersion UV source and magnetic stirrer. The low pressure mercury lamp TNN 15/32 (Heraeus, Hanau) with the main emission wavelength of $254 \mathrm{~nm}$ was applied. Actinometric experiments with uranyl oxalate and potassium ferric oxalate [17] gave the intensity of radiation inside the reactor of $2.49 \times$ $10^{18}$ quanta/s dm $\mathrm{dm}^{3}$. The study was performed for three selected compounds: benzo[a]pyrene (98\%, Sigma), chrysene (95\%, Fluka) and fluorene (Sigma) without additional purification. Stock solutions of PAHs were prepared in ethanol (p.a., 96\%, Polmos). Reaction solutions were done by introducing proper volume of ethanol solution (usually tens of $\mu \mathrm{l}$ ) into distilled water treated in a Millipore Milli-Q Plus System. Earlier suitable quantity of hydrogen peroxide (30\% POCh Gliwice) was added to water. The $\mathrm{pH}$ of reaction solution was adjusted by adding exact volume of natrium hydroxide (P.Ch. Lublin) or phosphoric acid (P.Ch. Lublin) solutions. As a radical scavenger tert-butyl alcohol (p.a., Fluka) was used. The samples of reaction solution for analysis were diluted with methanol (HPLC reagent, Baker Anal- 
ysed), so the final solvent composition was 50/50 vol. water/methanol. The addition of methanol stabilised the samples preventing PAHs deposition onto walls of flasks. The spectrofluorimetric analysis of reaction mixture was carried out [18] using Perkin Elmer LS50B apparatus. All experiments were performed at temperature $20^{\circ} \mathrm{C}$.

\section{RESULTS AND DISCUSSION}

3.1. Influence of parameters. The primary experiments were carried out without UV radiation to determine the participation of "dark" PAHs degradation under the influence of hydrogen peroxide at the concentration of $0.1 \mathrm{M}$. After $30 \mathrm{~min}$ of reaction time no depletion in BAP, CHR and FLU concentration was observed. After the next $10 \mathrm{~min} 20 \%$ of BAP and CHR converted into products, but FLU concentration remained unchanged. Other PAHs were also found to be recalcitrant towards oxidation by hydrogen peroxide alone $[14,16]$.

The next step of our study was focused on determination of the optimal $\mathrm{H}_{2} \mathrm{O}_{2}$ concentration in the reaction medium. For generation of hydroxyl radicals by hydrogen peroxide photolysis (1) a relatively high dosage of $\mathrm{H}_{2} \mathrm{O}_{2}$ is required. When comparing

$$
\mathrm{H}_{2} \mathrm{O}_{2} \stackrel{h v}{\longrightarrow} 2 \cdot \mathrm{OH}, \quad r_{1}=2 \phi_{\mathrm{H}} I_{a}
$$

to the $\cdot \mathrm{OH}$ radicals formation in the $\mathrm{O}_{3} / \mathrm{UV}$ system, the photolysis of hydrogen peroxide is about 50 times slower than that of ozone [19]. High $\mathrm{H}_{2} \mathrm{O}_{2}$ concentration in turn effectively quenches hydroxyl radicals (2) causing inhibition of next reactions [20].

$$
\mathrm{H}_{2} \mathrm{O}_{2}+{ }^{\bullet} \mathrm{OH} \rightarrow \mathrm{HO}_{2}^{\bullet}+\mathrm{H}_{2} \mathrm{O}, \quad r_{2}=k_{2} C_{\mathrm{OH}} C_{\mathrm{H}} .
$$

Figure 1 presents the evolution of BAP's relative concentration versus time for various hydrogen peroxide concentrations. The most effective conversion was observed for $\mathrm{H}_{2} \mathrm{O}_{2}$ content about $0.01 \mathrm{M}$ (Figure 2). The same results were obtained for the other two PAHs.

The different $\mathrm{pH}$ of reaction solution can influence the reaction rate changing on the one hand the acidbase equilibrium of $\mathrm{H}_{2} \mathrm{O}_{2}$ (3) and modifying target molecule conformation on the other hand.

$$
\mathrm{H}_{2} \mathrm{O}_{2} \longleftrightarrow \mathrm{H}^{+}+\mathrm{HO}_{2}^{-}, \quad \mathrm{pK}=11.6[19] .
$$

The latter induces among others, the alteration of extinction coefficient, which in our case was observed for CHR (Figure 3) and BAP. In acidic solutions a sharp increase of the absorption was noticed.

The degradation of PAHs was studied at three $\mathrm{pH}$ values: 2.5, 7 and 11.7. During reactions the changes of $\mathrm{pH}$ were not observed, probably due to very low concentrations of acidic products. Figure 4 presents the evolution in normalised CHR concentration during reaction for various $\mathrm{pH}$ values. The highest rate of conversion took place in neutral and next in acidic solutions. In alkaline mixture the reaction rate explicitly decreased. The same relation was observed for FLU, but for BAP

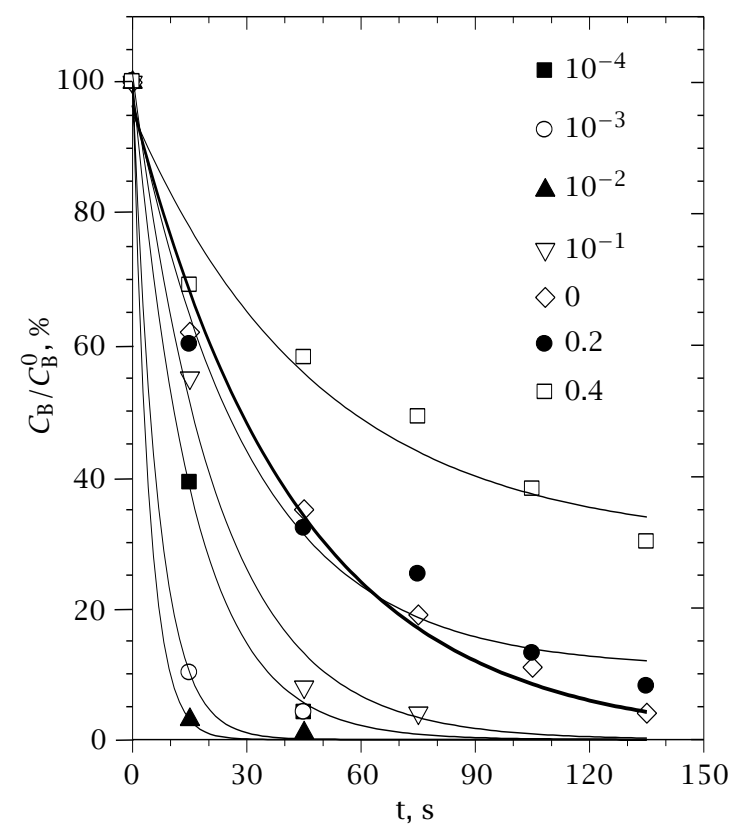

Figure 1. Evolution of relative BAP concentration versus time for various hydrogen peroxide concentrations; $C_{\mathrm{H}}^{0}, M$. $\mathrm{pH}=7, C_{\mathrm{B}}^{0}=4.76 \times 10^{-9} \mathrm{M}$.

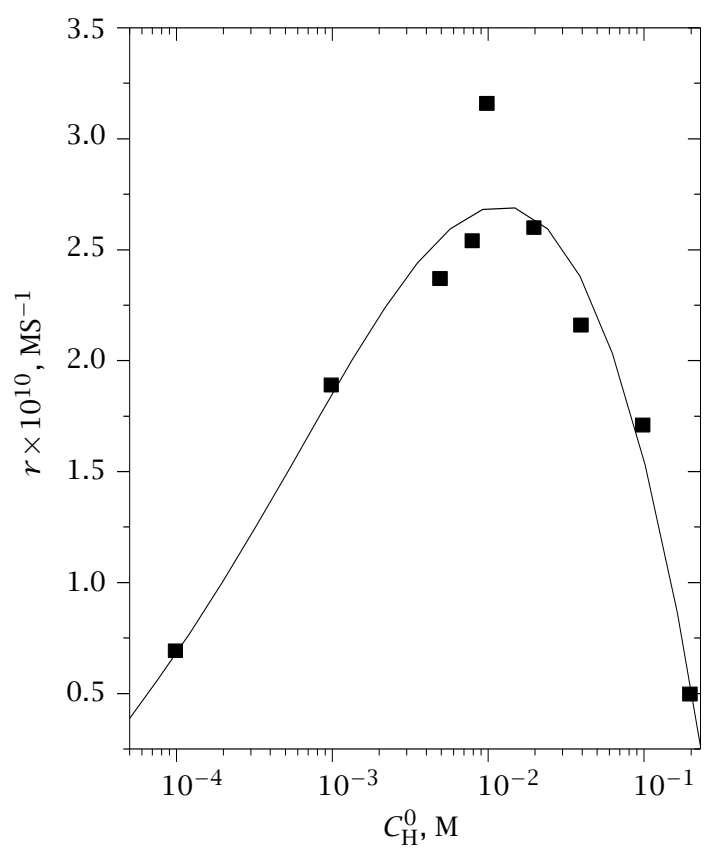

Figure 2. Dependence of CHR degradation rate on hydrogen peroxide concentration. $\mathrm{pH}=7, C_{\mathrm{C}}^{0}=5.12 \times 10^{-9} M$.

the best conditions for its degradation were noticed at $\mathrm{pH}=2.5$.

The PAHs in the $\mathrm{H}_{2} \mathrm{O}_{2} / \mathrm{UV}$ system disappear due to direct photolysis and to their reaction with hydroxyl radicals. In alkaline solution part of the light absorbed by hydrogen peroxide, exactly its ionic formhydroperoxide anion (3), increases since the extinction coefficient increases from $19 \mathrm{M}^{-1} \mathrm{~cm}^{-1}$ for $\mathrm{H}_{2} \mathrm{O}_{2}$ [21] to 


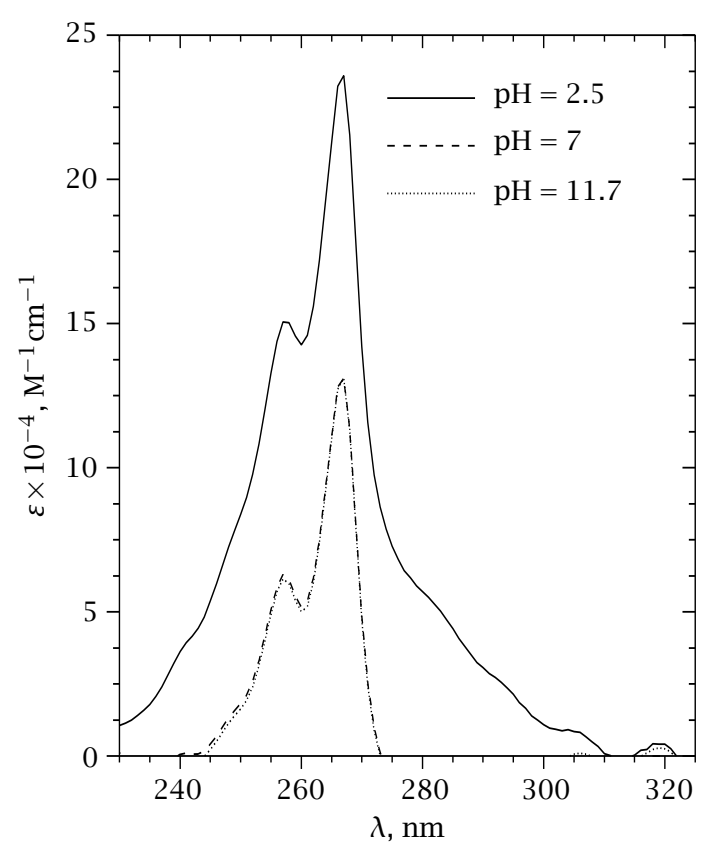

Figure 3. Absorption spectra of CHR for different $\mathrm{pH}$ in 50/50 vol. methanol/water solution $C_{C}=1.14 \times 10^{-6} \mathrm{M}$.

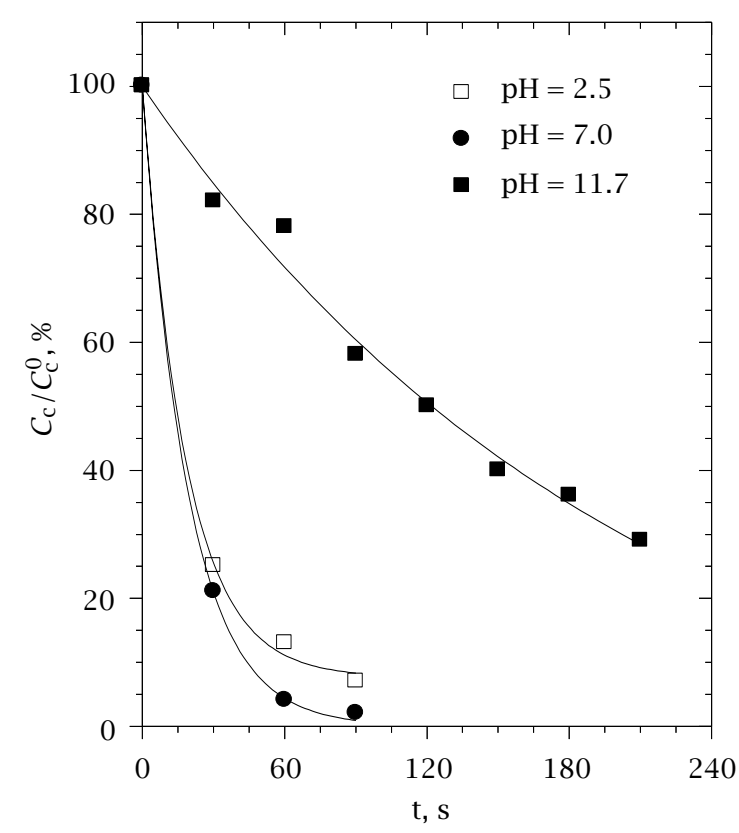

Figure 4. Variation of relative CHR concentration versus time in acidic, neutral and alkaline solutions. $C_{\mathrm{C}}^{0}=5.12 \times$ $10^{-9} M, C_{\mathrm{H}}^{0}=1 \times 10^{-3} M$.

$240 \mathrm{M}^{-1} \mathrm{~cm}^{-1}$ for $\mathrm{HO}_{2}^{-}$[11]. This diminishes the formation of hydroxyl radicals according to equation (1). Additionally, $\bullet$ OH radicals can be consumed not only in reaction (2) but also in the following reaction, which in these circumstances become very important.

$$
\mathrm{HO}_{2}^{-}+{ }^{\bullet} \mathrm{OH} \rightarrow \mathrm{HO}_{2}^{\bullet}+\mathrm{OH}^{-}, \quad r_{4}=k_{4} C_{\mathrm{OH}} C_{\mathrm{HO}} .
$$

Moreover, if the concentration of $\mathrm{H}_{2} \mathrm{O}_{2}$ is low, then part of the light absorbed by PAHs attains a consider- able value, BAP and CHR quench smaller light quantity as their extinction coefficient decreases at high $\mathrm{pH}$. So the reaction of direct photolysis is also retarded.

$$
\mathrm{PAH} \rightarrow \text { products, } \quad r_{5}=\phi_{\mathrm{P}} I_{\mathrm{a}} .
$$

In conclusion, when $\mathrm{pH}$ is increased the oxidation rate of PAHs in $\mathrm{H}_{2} \mathrm{O}_{2}$ /UV system decreases.

The study of the PAHs degradation using hydrogen peroxide and UV radiation was also performed with the addition of $t-\mathrm{BuOH}$, known as hydroxyl radical scavenger [22]. The decomposition rate of BAP and CHR decreased as the concentration of $t-\mathrm{BuOH}$ in reaction solution increased, which is shown in Figure 5. The relationship observed for radical reaction seems to be obvious, however in the case of FLU no influence of the scavenger on reaction rate is reported. The same phenomena for FLU, acenaphthene and phenanthrene were presented by Beltran et al. [14] in their work with the use of bicarbonate ion as a radical quencher. But their explanation is unapplicable to our case. Probably, the degradation of FLU occurred according to the mechanism other than the radical one. However, a detailed explanation requires more experimental data.

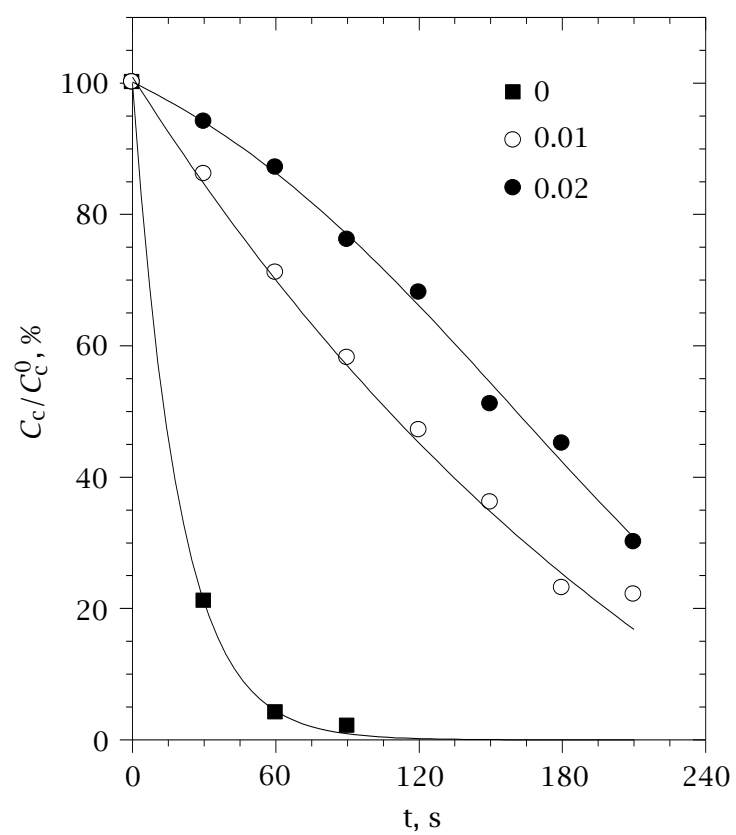

Figure 5. The influence of radical scavenger concentration $C_{\mathrm{t}}^{0}, M$ on the CHR degradation rate. $\mathrm{pH}=7, C_{\mathrm{C}}^{0}=$ $5.12 \times 10^{-9} M, C_{\mathrm{H}}^{0}=1 \times 10^{-3} M$.

3.2. Kinetic computing. The oxidation of PAHs by hydrogen peroxide combined with UV radiation can be treated as the process consisting of direct photolysis (5) and reaction of hydroxyl radicals:

$$
\mathrm{PAH}+{ }^{\bullet} \mathrm{OH} \rightarrow \text { products, } \quad r_{6}=k_{6} C_{\mathrm{OH}} C_{\mathrm{P}} .
$$

According to the above, the rate of this AOP can be written down as:

$$
r=-\frac{d C_{\mathrm{P}}}{d t}=r_{\mathrm{UV}}+k_{6} C_{\mathrm{OH}} C_{\mathrm{P}} .
$$


The rate of photolysis follows the Lambert-Beer law:

$$
r_{\mathrm{UV}}=\phi_{\mathrm{P}} I_{0} f_{\mathrm{P}}\left[1-\exp \left(-2.303 b \sum \varepsilon_{i} C_{i}\right)\right]
$$

where $f_{\mathrm{P}}$ is the fraction of radiation absorbed by a given PAH:

$$
f_{\mathrm{P}}=\frac{\varepsilon_{\mathrm{P}} C_{\mathrm{P}}}{\sum \varepsilon_{i} C_{i}} .
$$

When reaction is carried out at high $\mathrm{H}_{2} \mathrm{O}_{2}$ concentration it practically absorbs total light flux $\left(f_{\mathrm{H}}=1\right)$ and direct PAHs photolysis can be neglected. Simultaneously, the hydrogen peroxide concentration in the initial stage of reaction can be treated as constant $[14,16]$. For these conditions equation (7) simplifies to the form:

$$
-\frac{d C_{\mathrm{P}}}{d t}=k_{z} C_{\mathrm{P}} \quad \text { where } k_{z}=k_{6} C_{\mathrm{OH}} .
$$

$k_{z}$ is the pseudo-first order rate constant, so the plot of integrated form of equation (10) should follow a straight line in the coordinates system $\ln C_{\mathrm{P}}{ }^{0} / C_{\mathrm{P}}$-t. The verification of this relationship is shown in Figure 6.

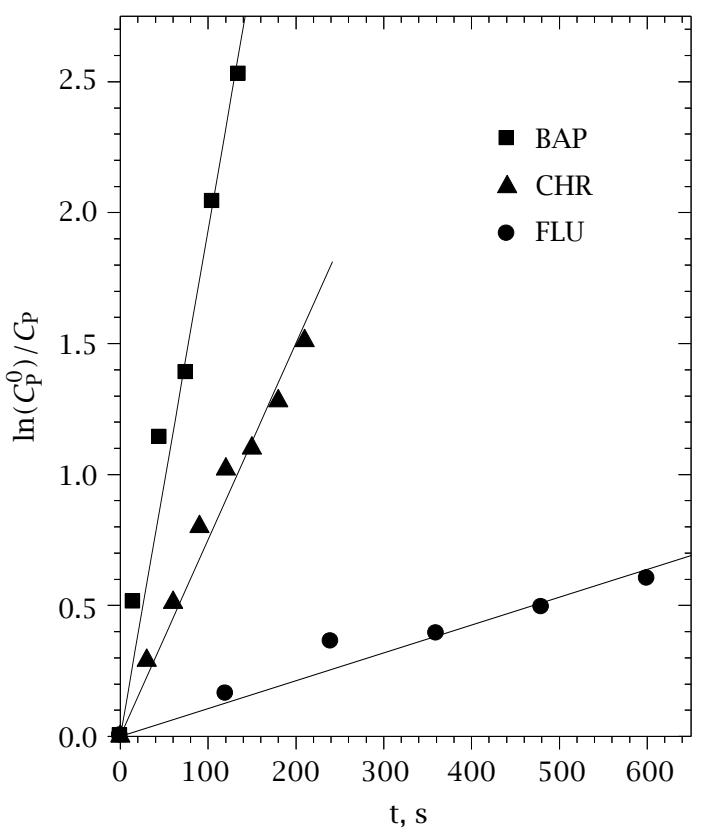

Figure 6. Determination of pseudo-first order rate constants $k_{z}$ (equation 10) of PAHs decomposition. $\mathrm{pH}=7$, $C_{\mathrm{B}}^{0}=4.76 \times 10^{-9} M, C_{\mathrm{C}}^{0}=5.12 \times 10^{-9} M, C_{\mathrm{H}}^{0}=0.2 M$ and $C_{\mathrm{F}}^{0}=5.42 \times 10^{-6} M, C_{\mathrm{H}}^{0}=0.4 M$.

Considering the reaction scheme described by reaction pathways (1), (2), (3), (4), (5) and (6) and assuming that other possible reactions of ${ }^{\bullet} \mathrm{OH}$ disappearance can be refused, the stationary state for hydroxyl radicals is described by $r_{1}=r_{2}+r_{4}+r_{6}$ and after expansion:

$$
\begin{aligned}
& 2 \phi_{\mathrm{H}} I_{0} f_{\mathrm{H}}\left[1-\exp \left(-2.303 b \sum \varepsilon_{i} C_{i}\right)\right] \\
& =k_{2} C_{\mathrm{OH}} C_{\mathrm{H}}+k_{4} C_{\mathrm{OH}} C_{\mathrm{HO}}+k_{6} C_{\mathrm{OH}} C_{\mathrm{P}} .
\end{aligned}
$$

This equation is useful in computing the concentration of hydroxyl radicals :

$$
C_{\mathrm{OH}}=\frac{2 \phi_{\mathrm{H}} I_{0} f_{\mathrm{H}}\left[1-\exp \left(-2.303 b \sum \varepsilon_{i} C_{i}\right)\right]}{k_{2} C_{\mathrm{H}}+k_{4} C_{\mathrm{HO}}+k_{6} C_{\mathrm{P}}} .
$$

The concentration of hydroperoxide anion $C_{\mathrm{HO}}$, which effectively quenches hydroxyl radicals (4) is pH dependent and it is calculated from the equilibrium constant (3):

$$
C_{\mathrm{HO}}=\frac{K C_{\mathrm{H}}^{0}}{\exp (-2.303 p H)+K} \quad \text { and } C_{\mathrm{H}}^{0}=C_{\mathrm{HO}}+C_{\mathrm{H}} \text {. }
$$

Combining equations (12) and (10) and determining $k_{z}$ (Figure 6) it is possible to calculate the rate constant of the reaction of PAH with hydroxyl radical. The values of $k_{2}=2.7 \times 10^{7} \mathrm{M}^{-1} \mathrm{~s}^{-1}$ and $k_{4}=7.5 \times 10^{9} \mathrm{M}^{-1} \mathrm{~s}^{-1}$ were taken from literature [20]. For studied PAHs the average rate constants $k_{6}$ were found to be: $2.53 \times 10^{10}$, $9.82 \times 10^{9}$ and $2.77 \times 10^{9} \mathrm{M}^{-1} \mathrm{~s}^{-1}$ for BAP, CHR and FLU, respectively.

Equation (12) permits to compute the concentration of hydroxyl radicals using determined $k_{6}$ rate constant. The selected results of our experiments and calculations are collected in Table 1. It is seen that hydroxyl radical concentration increases with an increase of hydrogen peroxide content to an optimal value, and then it falls down. In alkaline reaction mixture one can see the decrease of $\bullet \mathrm{OH}$ radicals concentration in relation to neutral or acidic solutions. The results of these calculations confirm the validity of the reaction model proposed.

\section{CONCLUSIONS}

The experiments performed on PAHs elimination from aqueous solution using $\mathrm{H}_{2} \mathrm{O}_{2}$ /UV system make it possible to draw the following conclusions.

According to literature data $[14,16,19]$ we found that beneficial doses of hydrogen peroxide which maximally accelerate the degradation equal to about $0.01 \mathrm{M}$ for all studied PAHs.

The rate of PAHs disappearance is $\mathrm{pH}$ dependent. The smallest rates were achieved in alkaline reaction mixtures.

The comparison of reactions carried out in the presence and absence of tert-butyl alcohol enables us to state that BAP and CHR decomposition follows radical reactions. In the case of FLU no influence of $t-\mathrm{BuOH}$ on reaction rate was observed which could indicate another pathway of its decomposition. The lack of inhibiting effect on the FLU degradation in the presence of bicarbonate ion was also noticed in the work of Beltran et al. [14].

The verified kinetic model, experimental and literature data enable computation of the rate constants of PAHs reaction with hydroxyl radicals. The obtained values are of the same order of magnitude as those cited in the literature $[14,15]$ and equal to $2.53 \times 10^{10}, 9.82 \times 10^{9}$ and $2.77 \times 10^{9} \mathrm{M}^{-1} \mathrm{~s}^{-1}$ for BAP, CHR and FLU, respectively. 
Table 1. The concentration of hydroxyl radicals $C_{\mathrm{OH}}$ computed according to equation (13)

\begin{tabular}{|c|c|c|c|c|c|}
\hline \multirow{2}{*}{ No. } & $C_{\mathrm{H}}^{0}$ & $r \times 10^{10}$ & $f_{\mathrm{H}}$ & $C_{\mathrm{HO}}$ & $C_{\mathrm{OH}} \times 10^{12}$ \\
\hline & $\mathrm{M}$ & $\mathrm{Ms}^{-1}$ & & $\mathrm{M}$ & M \\
\hline \multicolumn{2}{|c|}{ BENZO[A]PYRENE } & \multicolumn{2}{|c|}{$C_{\mathrm{B}}^{0}=4.76 \times 10^{-9} \mathrm{M}$} & \multicolumn{2}{|c|}{$k_{6}=2.53 \times 10^{10} \mathrm{M}^{-1} \mathrm{~s}^{-1}$} \\
\hline 1 & $10^{-3}$ & 6.51 & 0.988 & $1.58 \times 10^{-8}$ & 22.9 \\
\hline 2 & $10^{-2}$ & 7.18 & 0.988 & $1.58 \times 10^{-7}$ & 12.1 \\
\hline 3 & 0.2 & 1.16 & 0.999 & $3.16 \times 10^{-6}$ & 0.76 \\
\hline $4^{\mathrm{a}}$ & $10^{-3}$ & 2.00 & 0.988 & $4.99 \times 10^{-9}$ & 23.0 \\
\hline $5^{\mathrm{b}}$ & $10^{-3}$ & 0.63 & 0.988 & $4.42 \times 10^{-4}$ & 0.19 \\
\hline \multicolumn{2}{|c|}{ FLUORENE } & \multicolumn{2}{|c|}{$C_{\mathrm{F}}^{0}=5.42 \times 10^{-6} \mathrm{M}$} & \multicolumn{2}{|c|}{$k_{6}=2.77 \times 10^{9} \mathrm{M}^{-1} \mathrm{~s}^{-1}$} \\
\hline 6 & $10^{-3}$ & 1273 & 0.186 & $1.58 \times 10^{-8}$ & 10.6 \\
\hline 7 & $10^{-2}$ & 1380 & 0.696 & $1.58 \times 10^{-7}$ & 10.0 \\
\hline 8 & 0.2 & 162.6 & 0.958 & $3,16 \times 10^{-6}$ & 0.73 \\
\hline 9 & 0.4 & 90.30 & 0.989 & $6.32 \times 10^{-6}$ & 0.38 \\
\hline $10^{\mathrm{a}}$ & $10^{-3}$ & 1000 & 0.186 & $4.99 \times 10^{-9}$ & 10.6 \\
\hline $11^{\mathrm{b}}$ & $10^{-3}$ & 321.0 & 0.186 & $4.42 \times 10^{-4}$ & 0.13 \\
\hline \multicolumn{2}{|c|}{ CHRYSENE } & \multicolumn{2}{|c|}{$C_{\mathrm{C}}^{0}=5.125 \times 10^{-9} \mathrm{M}$} & \multicolumn{2}{|c|}{$k_{6}=9.81 \times 10^{9} \mathrm{M}^{-1} \mathrm{~s}^{-1}$} \\
\hline 12 & $10^{-3}$ & 1.88 & 0.985 & $1.58 \times 10^{-8}$ & 22.9 \\
\hline 13 & $10^{-2}$ & 3.15 & 0.998 & $1.58 \times 10^{-7}$ & 12.3 \\
\hline 14 & 0,2 & 0.48 & 0.999 & $3.16 \times 10^{-6}$ & 0.36 \\
\hline $15^{\mathrm{a}}$ & $10^{-3}$ & 1.74 & 0.985 & $4.99 \times 10^{-9}$ & 23.4 \\
\hline $16^{\mathrm{b}}$ & $10^{-3}$ & 0.27 & 0.985 & $4.42 \times 10^{-4}$ & 0.19 \\
\hline
\end{tabular}

${ }^{\mathrm{a}} \mathrm{pH}=2.5 ; \quad{ }^{\mathrm{b}} \mathrm{pH}=11.7$

\section{ACKNOWLEDGEMENTS}

The study was sponsored by the Polish Committee for Scientific Research, Grant No.3T09C 02412. The authors are grateful to the Foundation for Development of Polish Science for purchasing Perkin Elmer spectrofluorimeter LS50B.

\section{NOMENCLATURE}

$b$-light path

$C$-concentration

$f$-fraction of absorbed radiation

$I_{0}$-intensity of incident radiation

$I_{\mathrm{a}}$-intensity of absorbed radiation

$k$-rate constant

$k_{z}$-pseudo-first order rate constant

$K$-equilibrium constant

$r$-reaction rate

$t$-time

\section{Greek letters}

$\varepsilon$-extinction coefficient

$\lambda$-wavelength

$\Phi$-quantum yield

Subscripts

B-benzo[a]pyrene

C-chrysene

F-fluorene

H-hydrogen peroxide

HO-hydroperoxide anion

$\mathrm{OH}$-hydroxide radical

\section{P-PAHs}

t-tert-butyl alcohol

\section{Superscript}

0 -initial value

\section{REFERENCES}

[1] I. G. Karanassios, V. I. Georgakilas, E. S. Lahaniatis, and G. A. Pilidis, Fresenius Envir. Bull. 3 (1994), 511.

[2] A. Saber, G. Morel, L. Paturel, J. Jarosz, M. MartinBouyer, and M. Vial, Fresenius J. Anal. Chem. 339 (1991), 716.

[3] A. Eschenbach, M. Kästner, R. Bierl, G. Schaefer, and B. Mahro, Chemosphere 28 (1994), 683.

[4] K. Krengel-Rothensee, BioEngineering 9 (1993), 13.

[5] D. Maier, M. Maier, B. Lloyd, and I. P. Toms, Ozone Sci. \& Eng. 18 (1997), 517.

[6] B. Fouillet, P. Chambon, M. Castegnaro, and N. Weill, Bull. Environ. Contam. Toxicol. 47 (1991), 1.

[7] A. Kornmüller, M. Cuno, and U. Wiesmann, Wat. Sci. Tech. 35 (1997), 57.

[8] C. E. Cerniglia, Journal of Industrial Microbiology \& Biotechnology 19 (1997), 324.

[9] A. K. De, S. Bhattacharjee, and B. K. Dutta, Ind. Eng. Chem. Res. 36 (1997), 3607.

[10] D. W. Sundstrom, B. A. Weir, and H. E. Klei, Environ. Prog. 8 (1989), 6. 
[11] E. Tace, Etude cinetique de la degradation de chloroethanes et de s-triazines en milieux aqueux par irradiation UV en absence et en presence de peroxyde hydrogene, Ph.D. thesis, Universite de Poitiers, France, 1992.

[12] F. J. Beltran, G. Ovejero, and B. Acedo, Water Res. 27 (1993), 1013.

[13] M. E. Sigman, A. C. Buchanan III, and S. M. Smith, J. Adv. Oxid. Technol. 2 (1997), 415.

[14] F. J. Beltran, G. Ovejero, and J. Rivas, Ind. Eng. Chem. Res. 35 (1996), 883.

[15] M. Hautaniemi, J. Kallas, R. Munter, M. Trapido, and Y. Veressinina, vol. 82, p. 1, Lappeenranta University of Technology, Finland, 1997.

[16] T. Tuhkanen, Oxidation of Organic Compounds in Water and Waste Water with the Combination of
Hydrogen Peroxide and UV Radiation, Ph.D. thesis, University of Kuopio, Finland, 1994.

[17] S. L. Murov, I. Carmichael, and G. L. Hug, Handbook of Photochemistry, 2nd ed., 1993, M. Dekker.

[18] J. S. Miller, Anal. Chim. Acta. 388 (1999), 27.

[19] J. Hoigne, Chemistry of Aqueous Ozone and Transformation of Pollutants by Ozonation and Advanced Oxidation Processes, The Handbook of Environmental Chemistry (Berlin, Heidelberg) (J. Hrubec, ed.), Springer-Verlag, 1998.

[20] H. S. Christensen, H. Sehested, and H. Corfitzan, J. Phys. Chem. 86 (1982), 15.

[21] I. Nicole, J. De Laat, M. Dore, J. Duguet, and C. Bonnel, Water Res. 24 (1990), 157.

[22] J. Staehelin and J. Hoigne, Environ. Sci. Technol. 19 (1985), 1206. 


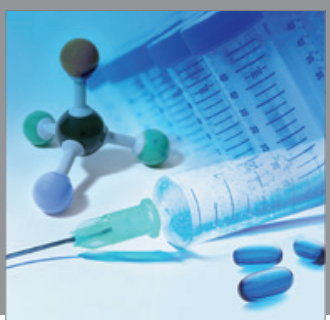

International Journal of

Medicinal Chemistry

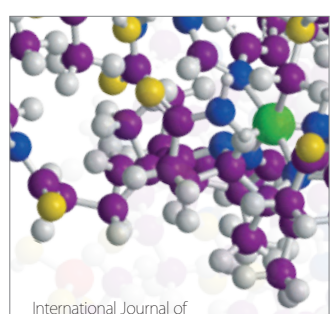

Carbohydrate Chemistry

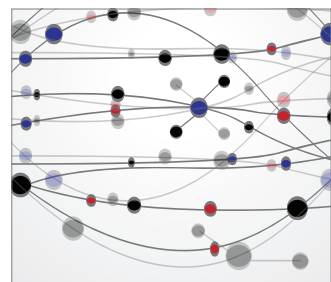

The Scientific World Journal
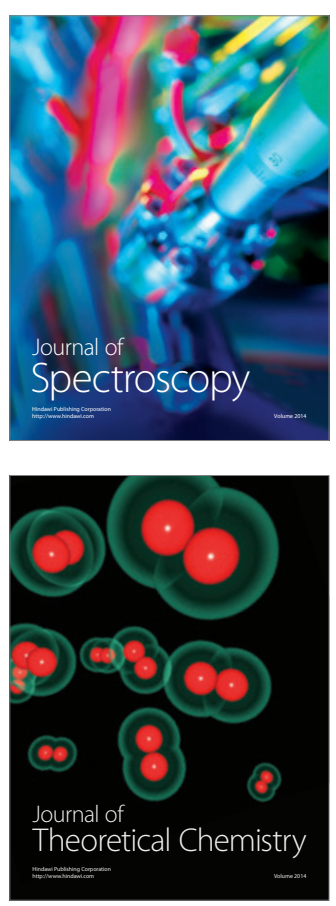
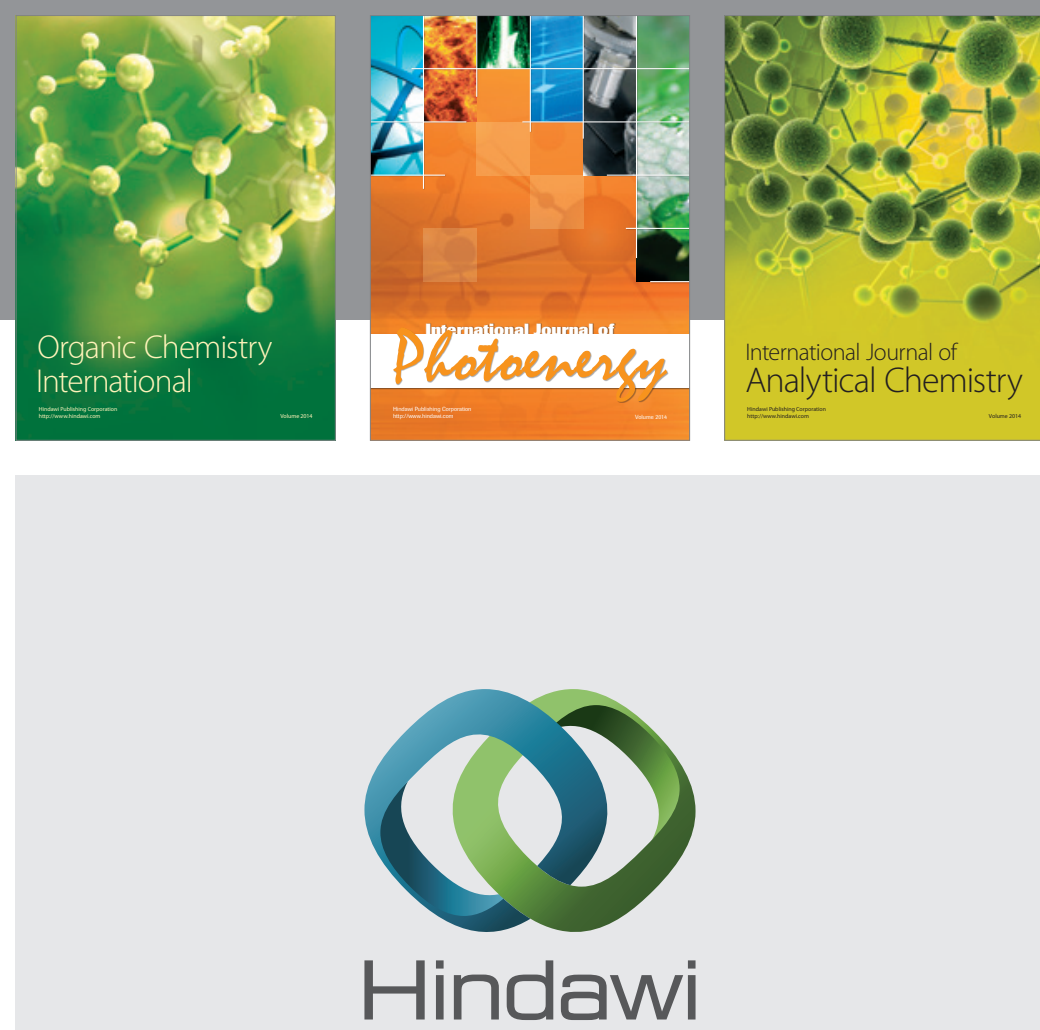

Submit your manuscripts at

http://www.hindawi.com
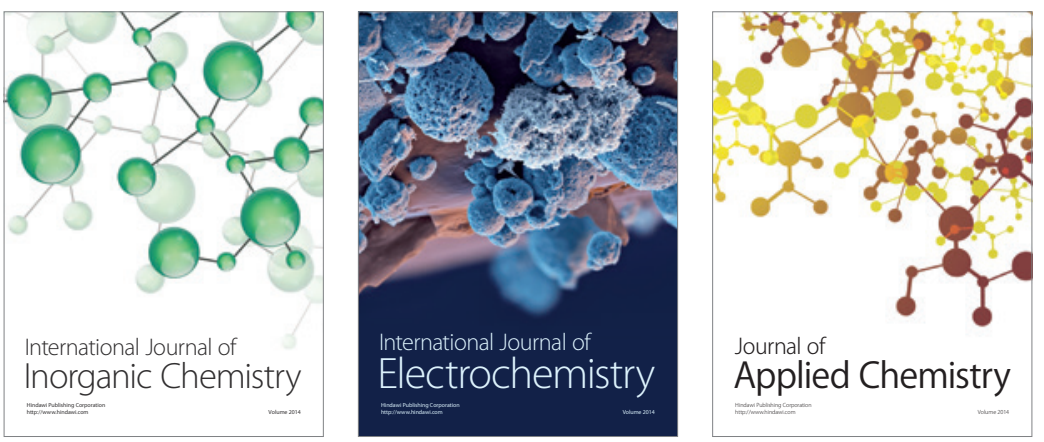

Journal of

Applied Chemistry
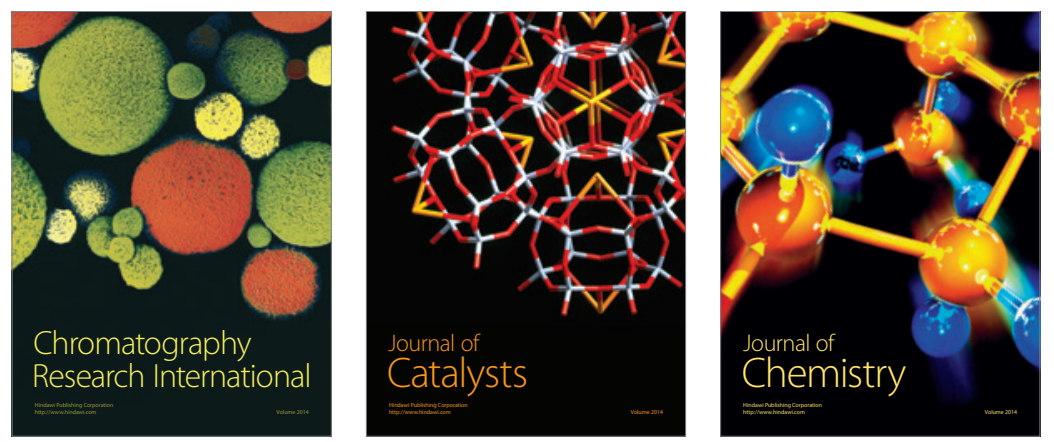
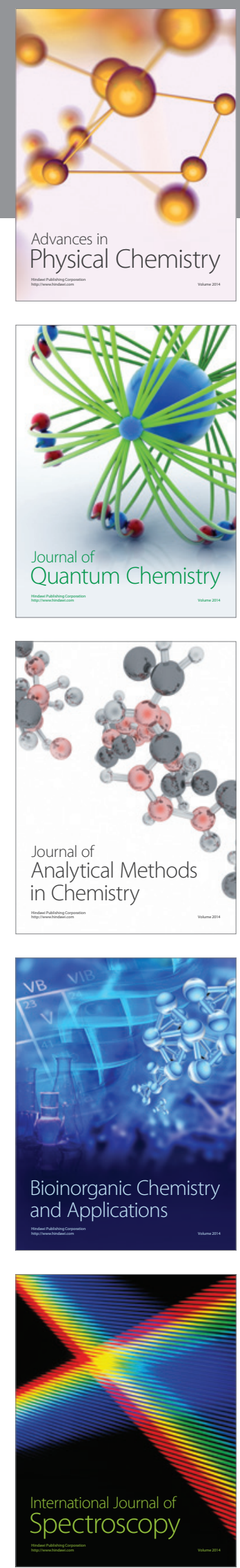